\title{
Effect of sildenafil citrate on prediabetic and diabetic albino rats treated with Metformin
}

\author{
Ahmad Taha', Haredy Hassan Haredy', Ahmed Ghareip ${ }^{3}$, Ahmed El-Sayed Nour El-Deen', \\ Yasser M Ashour ${ }^{4}$ \\ ${ }^{1}$ Department of Physiology, Faculty of Medicine Port Said University \\ ${ }^{2}$ Department of Pharmacology, Faculty of Medicine, Al-Azhar University, Assuit, Egypt. \\ ${ }^{3}$ Department of Physiology, Faculty of Medicine, Al-Azhar University, Assuit, Egypt. \\ ${ }^{4}$ Department of Physiology, Egyptian Russian University
}

\begin{abstract}
Diabetes Miletus (DM) is a global epidemic disease. It is estimated that there are already 415 million adults aged 2079 years diabetics worldwide. Sildenafil citrate is a phosphodiesterase type 5 (PDE5) inhibitor, which increases cyclic guanosine monophosphate (cGMP) and metformin (MET) is a biguanide used for the treatment of type 2 diabetes which increases peripheral insulin sensitivity. Aim: This study aims to assess the effect of sildenafil citrate and metformin on lipid profile and glycemic control in diabetic and prediabetic albino rats. Materials and methods: Adult male albino rats are used and divided into nine groups each group consists of 10 rats, diabetes is induced by feeding a high-fat $\operatorname{diet}($ HFD) for an initial period of 2 weeks followed by a single intraperitoneal injection of ( $35 \mathrm{mg} / \mathrm{kg}$ ) Streptozotocin. Prediabetes is induced by feeding (HFD) and glucose in water for a period of 2 weeks. Sildenafil was given in a dose of (5 $\& 10 \mathrm{mg} / \mathrm{kg} /$ day orally for 4 weeks), metformin was given in a dose of ( $50 \& 100 \mathrm{mg} / \mathrm{kg} /$ day orally for 4 weeks) using oral gavages to normal healthy rats, diabetic and prediabetic rats. Blood samples were collected after 4 weeks of treatment in all experimental groups. Results: Combined administration of sildenafil and metformin on diabetic rats improving hyperglycemia, oxidative stress, and hyperlipidemia induced by streptozotocin than the administration of metformin or sildenafil alone. Conclusion: Sildenafil has beneficial effects against some diabetic complications. The present study showed that sildenafil with metformin has beneficial effects against diabetic complications.
\end{abstract}

KEYWORDS: Diabetes Miletus; Sildenafil Citrate; Metformin; Prediabetic

\section{INTRODUCTION}

$\mathrm{D}$ iabetes Meletus (DM) is worldwide epidemic disease. It is estimated that there are about 415 million adults aged 20-79 with diabetes, including 193 million who are undiagnosed[1]. A further 318 million adults have impaired glucose tolerance, which puts them at high risk of developing the disease. By the end of 2015, If this high rate not stopped, by 2040 there will be 642 million people living with the disease [2]. It is estimated that $5 \%-10 \%$ of diagnosed cases are of Type I diabetes mellitus (DM), while the remaining $90 \%-95 \%$ are of Type II DM $[3,4]$.
Erectile dysfunction (ED) is defined as the persistent inability to attain and maintain an erection sufficient to permit satisfactory sexual activity and is a common complication of DM in men [4]. The prevalence of ED of any degree in men aged 40-70 years was predestined to be $52 \%$. ED occurs at an earlier age in men with DM than in men in the general population [5]. ED in diabetes is often complex and caused by several mechanisms including vascular disease, autonomic neuropathy, hormonal imbalance, and psychogenic factors. The risk of ED increases with duration of diabetes and is directly related to poor glycemic control and the presence of other risk factors such as hypertension, smoking, and

Carrespondence: Ahmed El-Sayed Nour El-Deen, Department of physiology, Faculty of Medicine, Al-Azhar University, Assuit, Egypt. E-mail: drnoor83@hotmail.com

eISSN: 2523-6709

pISSN: 2523-6695

DOI: 10.31878/ijcrpp.2021.51.02
(C) Authors; 2021. (CC BY-NC-SA 4.0)

This is an Open Access article which permits unrestricted

non-commercial use, provided the original work is properly cited. 
hyperlipidemia [4].

Treatment options for ED include psychological management, vacuum constriction devices, intracavernosal injections, transurethral drug delivery, penile prostheses, vascular surgery, and modification of medication contributing to the problem [6]. However, many of these treatments have limited acceptability to users [7]. The ideal goal in the treatment of ED is restoration of erectile capacity using a minimally invasive and safe treatment [8]. Sildenafil is the first oral drug to be marketed specifically for the treatment of ED [4]. Sildenafil has the potential to enhance erectile activity under conditions of sexual stimulation. It does not have a direct effect on libido or smooth muscle [6].

The use of Sildenafil in patients with DM in highly increased and its effect on glycated hemoglobin and fasting plasma glucose are still inconclusive [9].

This study is an attempt to assess the effect of daily oral sildenafil citrate on lipid profile and glycemic control in diabetic and prediabetic rats with metabolic syndrome, whether Sildenafil might represent new therapeutic approaches for preventing and treating metabolic syndrome.

\section{Material and Methdos}

This work was done at faculty of medicine, AlAzhar University (Assiut branch), pharmacology department.

Experimental animals: Ninety adult male albino rats were chosen as an animal model for this study with initial body weight (BW) ranging from 160-180 g. Rats were purchased from animal house, Faculty of Medicine, Assiut University, Assiut, Egypt, and were maintained on standard diet of known composition, and water supply freely in clean containers. They were kept for two weeks to adapt to the laboratory conditions before the start of the experiment. The animals were housed in standard polypropylene cages with stainless steel good, aerated covers and maintained under controlled room temperature with 12 hrs light-dark cycle.

Induction of diabetes: Type $2 \mathrm{DM}$ was induced by feeding a high fat diet (HFD) for 2 weeks followed by an intraperitoneal injection of $35 \mathrm{mg} / \mathrm{kg}$ b.wt streptozotocin dissolved in citrate buffer $\mathrm{pH} 4.5$ [10]. After that, the rats were kept for next 48 hours on oral $10 \%$ glucose solution on top of their chaw to prevent hypoglycemia [11]. After Seven days the blood glucose levels for injected rats were measured. Rats having blood glucose level $\geq 200 \mathrm{mg} / \mathrm{dl}$, after 2 hours of glucose intake were considered diabetic [10].

Induction of prediabetes: Prediabetes in rats were induced by feeding a high fat diet (HFD) (45\% energy from fat) and glucose in water for period of 2 weeks. Development of prediabetes was confirmed by development of obesity, hyperinsulinemia, and insulin resistance and not frank hyperglycemia or DM, in these cases the rats considered prediabetic [12] .

Experimental design: The experimental animal was divided into nine groups, each group comprising ten rats designated as follows:

\section{G1: Normal control rats.}

\section{G2: Diabetic control rats.}

G3: Diabetic rats treated with metformin $100 \mathrm{mg} / \mathrm{kg}$ b.wt orally for 4 weeks.

G4: Diabetic rats treated with Sildenafil citrate $10 \mathrm{mg} / \mathrm{kg}$ b.wt orally for 4 weeks.

G5: Diabetic rats treated with Sildenafil citrate $10 \mathrm{mg} / \mathrm{kg}$ b.wt and metformin $100 \mathrm{mg} / \mathrm{kg}$ b.wt orally for 4 weeks.

G6: Prediabetic control rats.

G7: Prediabetic rats treated with metformin 50 $\mathrm{mg} / \mathrm{kg}$ b.wt orally for 4 weeks.

G8: Prediabetic rats treated with Sildenafil citrate $5 \mathrm{mg} / \mathrm{kg}$ b.wt orally for 4 weeks.

G9: Prediabetic rats treated with Sildenafil citrate $5 \mathrm{mg} / \mathrm{kg}$ b.wt and metformin $50 \mathrm{mg} / \mathrm{kg}$ b.wt orally for 4 weeks.

Collection of samples for analysis: At the end of the 6th week, food was withdrawn from the rats and they were fasted for $8 \mathrm{~h}$, but had free access to water, and then anesthetized with thiopental (40 mg/kg ip) [13]. Blood samples were collected from orbital venous plexus in non-heparinized tubes. Blood samples were centrifuged at 2500 $\mathrm{rpm}$ for $15 \mathrm{~min}$, and the clear samples of blood serum were separated and stored at $-80 \circ \mathrm{C}$ until used for the measurement of the levels of blood glucose (BG) level (mg/dl), insulin level $(\mathrm{pmol} / \mathrm{L})$, total cholesterol (TC) (mg/dl), LDLcholesterol (mg/dl), HDL-cholesterol (mg/dl) and triglycerides (TG) (mg/dl).

Biochemical studies: Serum glucose level was measured by glucose oxidase method using Blood glucose (BG) kit (Egyptian Company for Biotechnology-Egypt) [14].

Insulin estimated using enzyme linked immunosorbent assay ELISA methods using Insulin kit (Sigma-Aldrich Co. LLC-USA) [15].

Serum cholesterol level was done by enzymatic -colometric test with lipid clearing factor) using Serum cholesterol kit (Egyptian company for biotechnology-Egypt) [16]. Serum triglycerides were estimated by an enzymatic colorimetric test with GPO-PAP method using Serum triglycerides kit (Egyptian Company for Biotechnology-Egypt) [17]. Serum high density lipoprotein (HDL) was estimated by enzymatic colorimetric method Using Serum high density lipoprotein (HDL) kit (Egyptian Company for Biotechnology-Egypt) 
[17].The serum LDL-cholesterol was estimated according to using the following equation; LDL-cholesterol in $\mathrm{mg} / \mathrm{dl}=$ Total cholesterol Triglyceride/5-HDL-cholesterol [17].

Statistical analysis: The obtained data were subjected to analysis of variance according to the procedures out lined by Snedecor and Cochran. The mean values were compared according to Duncan's multiple range test (DMRT) [18]. The data were analysed using Co Stat software for windows (version 6.3), (Co Hort Software, Monterey, Calif).

\section{RESULTS}

Changes of BW, BG, and insulin level among different groups.

A-In diabetic groups: The obtained data showed a significant $(\mathrm{p}<0.01)$ increase of body weight in G4 than diabetic control group with no significant $(\mathrm{P}>0.05)$ change between G3, G5 and diabetic control group as shown in table (1) and figure (1). The BG level showed a significant $(\mathrm{p}<0.01)$ lower level in all diabetic treated groups than control diabetic group with significant $(\mathrm{p}<0.01)$ lower level of G5 than G4 and G3 groups as shown in table (1). There was significant $(p<0.01)$ increase of serum insulin level in G4 and G5 than diabetic control group with no significant $(\mathrm{P}>0.05)$ change between $\mathrm{G} 3$ and diabetic control group as shown in table 1 .

Table 1. Changes of body weight, blood glucose and insulin level among different groups.

\begin{tabular}{|l|c|c|c|}
\hline Gr & B w (g) & BG (mg/dl) & Insulin(IU/dl) \\
\hline G1 & $170.2 \mathrm{c} \pm 12.7$ & $89.3 \mathrm{~d} \pm 12.5$ & $12.7 \mathrm{c} \pm 2.2$ \\
\hline G2 & $134 \mathrm{e} \pm 15.59$ & $230 \mathrm{a} \pm 35.8$ & $4.17 \mathrm{e} \pm 0.4$ \\
\hline G3 & $131.8 \mathrm{e} \pm 12.6$ & $119.7 \mathrm{~b} \pm 14.3$ & $4.87 \mathrm{e} \pm 0.9$ \\
\hline G4 & $155.2 \mathrm{~d} \pm 8.7$ & $124.2 \mathrm{~b} \pm 16.3$ & $10.1 \mathrm{~d} \pm 1.1$ \\
\hline G5 & $137.3 \mathrm{e} \pm 12.8$ & $110.2 \mathrm{c} \pm 7.3$ & $10.6 \mathrm{~d} \pm 0.8$ \\
\hline G6 & $197.3 \mathrm{a} \pm 10.4$ & $110.7 \mathrm{c} \pm 8.6$ & $15.2 \mathrm{~b} \pm 2.9$ \\
\hline G7 & $178.3 \mathrm{~b} \pm 10.6$ & $98.7 \mathrm{~d} \pm 11.1$ & $15.92 \mathrm{~b} \pm 2.6$ \\
\hline G8 & $201.8 \mathrm{a} \pm 10.7$ & $98.5 \mathrm{~d} \pm 7.7$ & $25.1 \mathrm{a} \pm 2.85$ \\
\hline G9 & $194.3 \mathrm{ab} \pm 7.2$ & $91.3 \mathrm{~d} \pm 5.5$ & $25.3 \mathrm{a} \pm 3.6$ \\
\hline F & $117.65^{\star *}$ & $180.2{ }^{* *}$ & $282.4^{* *}$ \\
\hline P. & 0.00 & 0.00 & 0.00 \\
\hline
\end{tabular}

Where G1: Control untreated; G2: Diabetic untreated rats; G3: Diabetic rats treated with metformin rats; G4: Diabetic rats treated with Sildenafil citrate; G5:Diabetic rats treated with Sildenafil citrate \& metformin; G6: Prediabetic untreated rats; G7: Prediabetic rats treated with metformin; G8:Prediabetic rats treated with Sildenafil citrate; G9: Preiabetic rats treated with Sildenafil citrate \& metformin.

** indicate $\mathrm{P}<0.01$. Means followed by a same letter are not significantly different at the $1 \%$ level by DMRT.F: F -test, P: p-value -letter (a) refer to highest mean and letter (b) referred to subsequent lower mean and so on.

Comparison between body weight percentages in all groups.

B-In prediabetic groups: The obtained data showed a significant $(\mathrm{p}<0.01)$ reduction of body weight in G7 than prediabetic control group with no significant $(\mathrm{P}>0.05)$ change between G8, G9 and prediabetic control group as shown in table (1). There was significant $(\mathrm{p}<0.01)$ lower level in Serum blood glucose level in all prediabetic treated groups than prediabetic control group with no significant $(\mathrm{P}>0.05)$ difference between all treated groups as shown in table (1). The serum insulin level showed a significant $(\mathrm{p}<0.01)$ increase in G8 and G9 than prediabetic control group with no significant $(\mathrm{P}>0.05)$ change between G7 and prediabetic control group as shown in table 1 .

The obtained data from fig (1-A) showed that in G3 body weight decreased by $2 \%$ but in G4 and G5 body weight increased by $16 \%$ and $3 \%$ respectively. The fig (1-D) showed that G3, G4 and G5 glucose level decreased by $48 \%, 46 \%$ and $52 \%$ respectively. Fig (1-E) showed that G3, G4 and G5 serum insulin level increased by $17 \%$, $142 \%$ and $154 \%$ respectively.

B-In prediabetic groups: The control prediabetic group value was calculated a $100 \%$ to compare between it and all treated groups.

The obtained data from fig (1-B) showed that G7 and G9 body weight decreased by $10 \%$ and $2 \%$ but in G8 body weight increased by $2 \%$ respectively. Data from fig (1-E) showed that G7, G8 and G9 glucose level decreased by $11 \%$, $11 \%$ and $17 \%$ respectively. Fig (1-F) showed that G7, G8 and G9 serum insulin level increased by $5 \%$, $65 \%$ and $67 \%$ respectively.

Changes of serum cholesterol, triglycerides, HDL and LDL level among different groups.

A-In diabetic groups: The obtained data showed a significant $(\mathrm{p}<0.01)$ lower level in Serum cholesterol in all diabetic treated groups than control diabetic group with significant $(\mathrm{p}<0.01)$ lower level of G5 than G3 and G4 groups as shown in table (2). There was a significant $(p<0.01)$ lower level in Serum triglycerides in all diabetic treated groups than control diabetic group with significant $(\mathrm{p}<0.01)$ lower level of G5 than G3 and G4 groups as shown in table (2). Serum HDL was significantly $(p<0.01)$ increased in all diabetic treated groups than control diabetic group with significant $(p<0.01)$ increase level of G5 than G3 and G4 groups as shown in table (2). The obtained data showed a significant $(p<0.01)$ lower level in Serum LDL in all diabetic treated groups than control diabetic group with significant $(\mathrm{p}<0.01)$ lower level of G5 than G3 and G4 groups as shown in table 2 . 

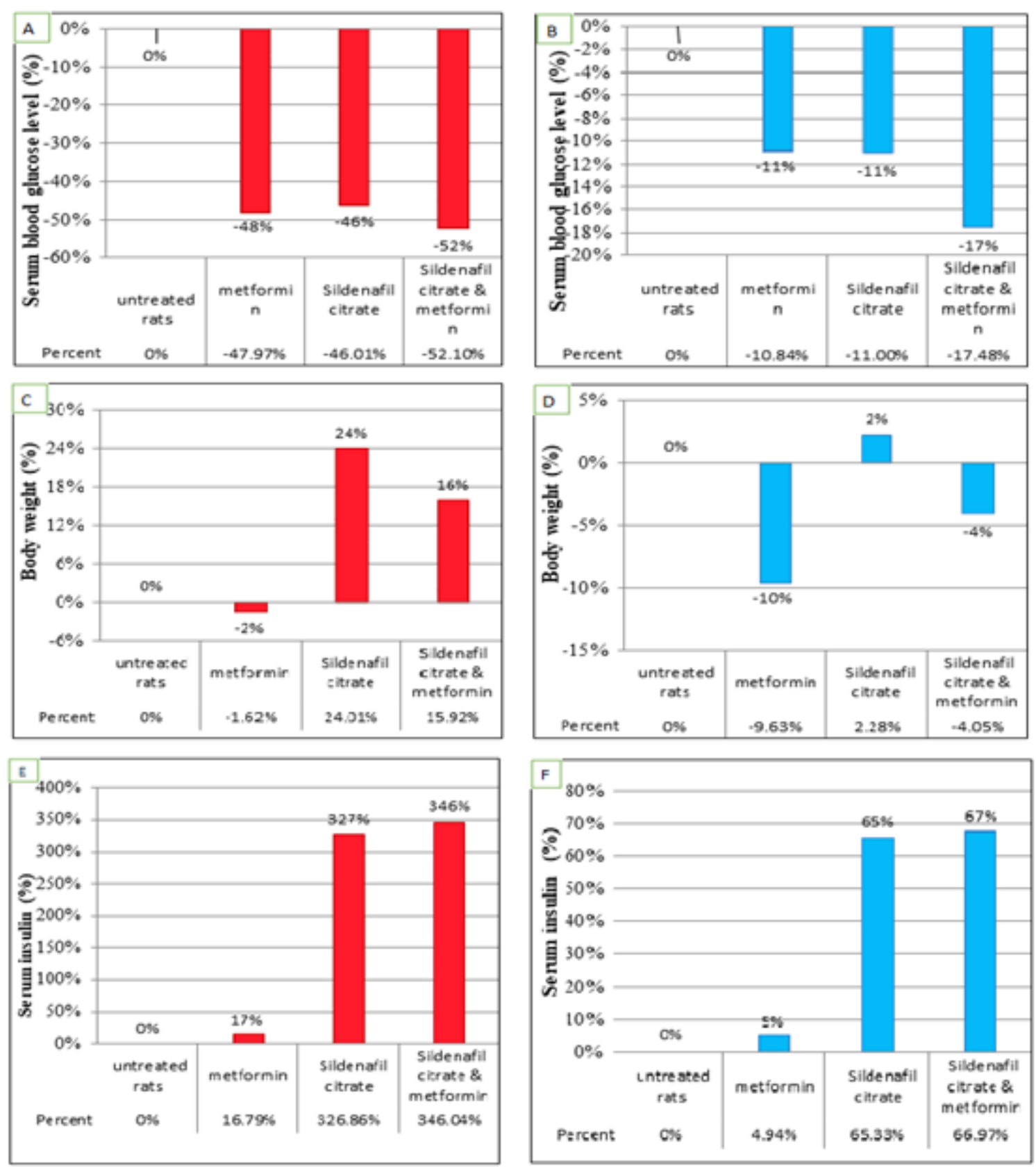

Diabetic rats groups

Non-Diabetic rats groups

Fig. (1): Percentage of body weight, Blood glucose and insulin level for different diabetic and prediabetic rats treated groups compare to diabetic and prediabetic untre ated groups.

Table 2. Changes of serum cholesterol, triglycerides, HDL and LDL level among different groups

\begin{tabular}{|c|c|c|c|c|}
\hline Gr & $\begin{array}{c}\text { Cholesterol } \\
(\mathrm{mg} / \mathrm{dl})\end{array}$ & TG $(\mathrm{mg} / \mathrm{dl})$ & $\begin{array}{c}\mathrm{HDL} \\
(\mathrm{mg} / \mathrm{dl})\end{array}$ & $\begin{array}{c}\mathrm{LDL} \\
(\mathrm{mg} / \mathrm{dl})\end{array}$ \\
\hline G1 & $66 \mathrm{~h} \pm 2.8$ & $50.5 \mathrm{~h} \pm 3.7$ & $37 \mathrm{a} \pm 2.8$ & $20 \mathrm{i} \pm 2.8$ \\
\hline G2 & $187.7 \mathrm{a} \pm 6$ & $141.8 \mathrm{a} \pm 4.6$ & $22 \mathrm{f} \pm 2.8$ & $139 \mathrm{a} \pm 5.2$ \\
\hline G3 & $88.8 \mathrm{~d} \pm 3$ & $70.8 \mathrm{e} \pm 2.9$ & $30.17 \mathrm{~d} \pm$ & $76.1 \mathrm{c} \pm 4.3$ \\
\hline G4 & $93.5 \mathrm{c} \pm 3.7$ & $98.3 \mathrm{c} \pm 4.3$ & $29.3 \mathrm{~d} \pm 3.5$ & $47.3 \mathrm{e} \pm 5.9$ \\
\hline G5 & $81.3 \mathrm{e} \pm 4.3$ & $65.2 \mathrm{f} \pm 2.9$ & $34 \mathrm{~b} \pm 2.8$ & $40.3 \mathrm{f} \pm 4.1$ \\
\hline G6 & $117.8 \mathrm{~b} \pm 6$ & $115.3 \mathrm{~b} \pm 4.3$ & $25.8 \mathrm{e} \pm 2.9$ & $81 \mathrm{~b} \pm 6.9$ \\
\hline G7 & $74.5 \mathrm{~g} \pm 3.7$ & $64.5 \mathrm{f} \pm 3.7$ & $32 \mathrm{c} \pm 2.8$ & $53.3 \mathrm{~d} \pm 4.3$ \\
\hline
\end{tabular}

\begin{tabular}{|c|c|c|c|c|}
\hline G8 & $78.2 \mathrm{f} \pm 5.7$ & $74.2 \mathrm{~d} \pm 5.3$ & $30.6 \mathrm{~cd} \pm 2.4$ & $36.2 \mathrm{~g} \pm 6.4$ \\
\hline G9 & $68.2 \mathrm{~h} \pm 5.7$ & $55.83 \mathrm{~g} \pm 4.6$ & $34.8 \mathrm{~b} \pm 2.9$ & $30.8 \mathrm{~h} \pm 4.6$ \\
\hline F & $1585^{\star \star}$ & $1303.7^{\star *}$ & $60.6^{\star *}$ & $1223.5^{\star *}$ \\
\hline P. & 0.00 & 0.00 & 0.00 & 0.00 \\
\hline
\end{tabular}

Where G1: Control untreated; G2: Diabetic untreated rats; G3: Diabetic rats treated with metformin rats; G4: Diabetic rats treated with Sildenafil citrate; G5:Diabetic rats treated with Sildenafil citrate \& metformin; G6: Prediabetic untreated rats; G7: Prediabetic rats treated with metformin; G8:Prediabetic rats treated with Sildenafil citrate; G9: Prediabetics rats treated with Sildenafil citrate \& metformin. 

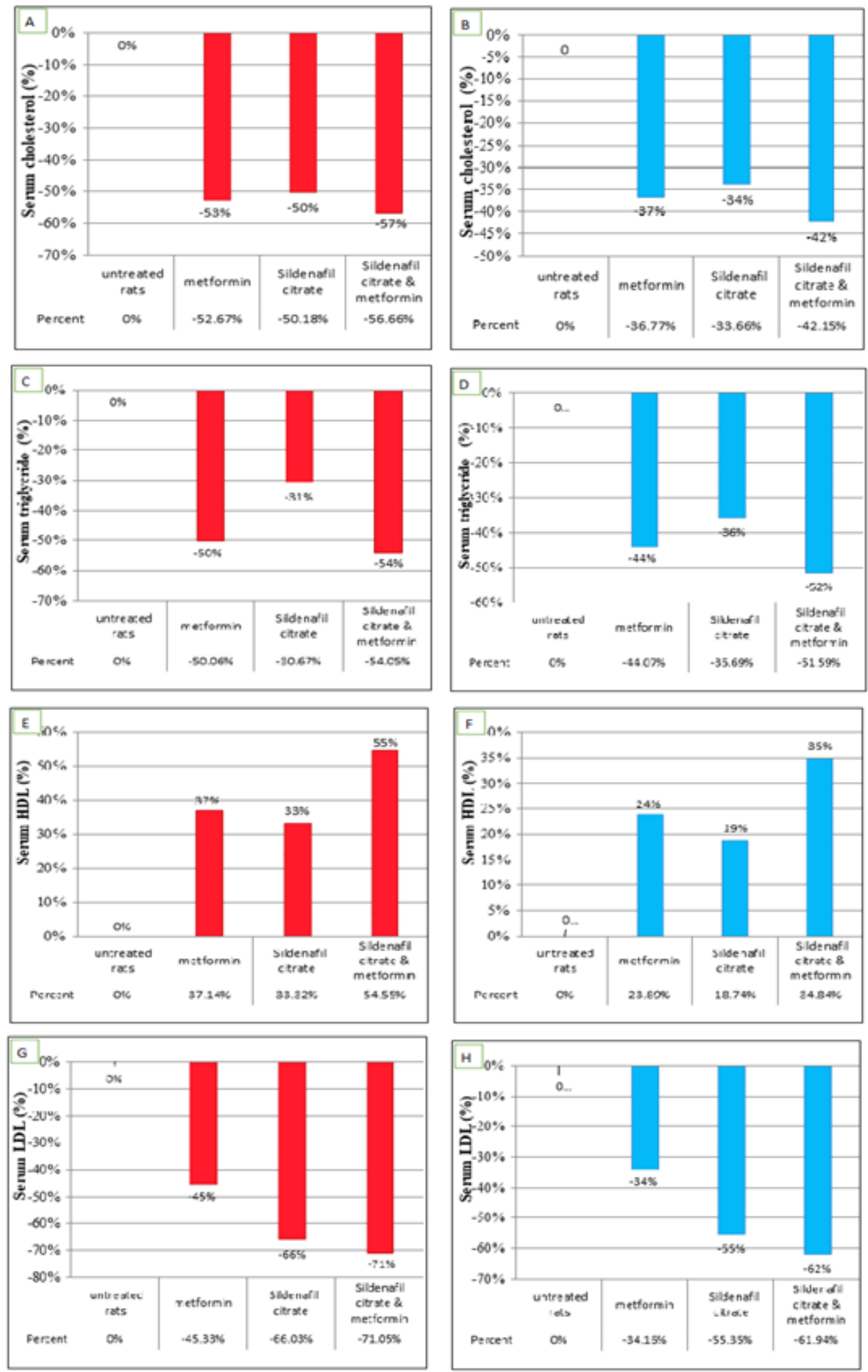

Diabetic rats groups

Non-Diabetic rats groups

Fig (2): Comparison between serum cholesterol, triglycerides, HDL and LDL level for different diabe tic and prediabetic rats treated groups compare to diabetic and prediabe tic untreated groups 
** indicate $\mathrm{P}<0.01$. Means followed by a same letter are not significantly different at the $1 \%$ level by DMRT.

-letter (a) refer to highest mean and letter (b) referred to subsequent lower mean and so on.

B-In prediabetic groups: The obtained data showed a significant $(\mathrm{p}<0.01)$ lower level in Serum cholesterol in all prediabetic treated groups than control prediabetic group with significant $(p<0.01)$ lower level of G9 than G7 and G8 groups as shown in table (2). There was significant $(p<0.01)$ lower level in Serum triglycerides in all prediabetic treated groups than control prediabetic group with significant $(p<0.01)$ lower level of G9 than G7 and G8 groups as shown in table (2). The HDL level showed a significant $(\mathrm{p}<0.01)$ increase in all prediabetic treated groups than control prediabetic group with significant $(\mathrm{p}<0.01)$ increase level of G9 than G7 and G8 groups as shown in table (2). The obtained data showed a significant $(p<0.01)$ lower level in Serum LDL in all prediabetic treated groups than control prediabetic group with significant $(p<0.01)$ lower level of G9 than G7 and G8 groups as shown in table 2.

Comparison between serum cholesterol, triglycerides, HDL and LDL level among different groups.

A-In diabetic groups: The control diabetic group value was calculated a $100 \%$ to compare between it and all treated groups.

The obtained data from fig (2-B) showed that G3, G4 and G5 serum cholesterol level decreased by $53 \%, 50 \%$ and $57 \%$ respectively. Fig (2-D) showed that G3, G4 and G5 serum triglycerides level decreased by $50 \%, 31 \%$ and $54 \%$ respectively. Serum HDL showed that G3, G4 and G5 level increased by $37 \%, 33 \%$ and $55 \%$ respectively fig $(2-F)$. There was decreased in serum LDL level in G3, G4 and G5 by $45 \%, 66 \%$ and $71 \%$ respectively as shown in fig $(2-\mathrm{H})$.

B-In prediabetic groups: The control prediabetic group value was calculated a $100 \%$ to compare between it and all treated groups. Fig (2-A) showed that G7, G8 and G9 serum cholesterol level decreased by $37 \%, 34 \%$ and $42 \%$ respectively. Serum triglycerides level in G7, G8 and G9 decreased by $44 \%, 36 \%$ and $52 \%$ respectively Fig (2-D).

The obtained data from fig (2-E) also showed that G7, G8 and G9 serum HDL level increased by $24 \%, 19 \%$ and $35 \%$ respectively and serum LDL level in G7, G8 and G9 decreased by 34\%, $55 \%$ and $62 \%$ respectively as shown in fig $(2-G)$.

\section{Discussion}

The results present study showed that administration of sildenafil citrate to prediabetic and diabetic albino rats treated with metformin has no significant changes on BW. The BW of diabetic rats treated with metformin by decreased by $2 \%$ and in diabetic rats treated with Sildenafil citrate and (Sildenafil citrate \& metformin) by $16 \%$ and $3 \%$ respectively in comparison with diabetic untreated rats as shown in fig (1). Body weight decreased by $10 \%$ and $2 \%$ in prediabetic rats treated with metformin and (Sildenafil citrate $\&$ metformin) but in prediabetic rats treated with Sildenafil citrate body weight increased by $2 \%$ respectively in comparison with prediabetic untreated rats as shown in fig (1). In the diabetic and prediabetic rats treated with metformin, the results of our work are in agreement with the results obtained by Mogul et al., 2003 [19] and Mamikutty et al [4] who reported that weight loss in obese women who were treated by metformin for 6 months was $5 \%$. In the diabetic and prediabetic rats treated with Sildenafil citrate, the results of our work agree with the results obtained by Johann et al. $\&$ Dilworth et al. \& Nisoli et al. who found that there is no effect of Sildenafil citrate on body weight $[8,20,21]$.

In the diabetic and prediabetic rats treated with metformin and sildenafil, there is no significant change $(\mathrm{P}<0.05)$ on body weight in comparison with control diabetic and prediabetic rats. Our results are in agreement with Bruckbauer et al. who reported that combination of metformin and sildenafil did not change body weight [22]. Tran and Howes found that Sildenafil citrate did not give any change in body weight of STZ diabetic induced rats and Chronic phosphodiesterase(PDE)-5 inhibitors lead to increase energy expenditure, that improve energy balance and body weight reduction that may enhance insulin action [23].

To explain this, it should be noted that increasing cGMP is a fundamental mechanism of action of sildenafil citrate [24]. A study on rat hepatocytes cGMP indicated that sildenafil citrate increases hepatocytes in vitro level but does not significantly alter glycogenolysis and gluconeogenesis [25]. Nitric oxide has been suggested as a second messenger molecule for the stimulatory effect of insulin in carbohydrate metabolism [26].

Mitschke et al. found that, chronic treatment (12 weeks) with the PDE-5 inhibitor sildenafil improved insulin sensitivity [27]. Lizhi, reported that PDE 5-inhibitors, lead to increase SIRT1 signaling in mice that lead to increase energy expenditure in skeletal muscle cells, improve energy balance and increase insulin action in both diet-induced obese, insulin resistant mice and diabetic patients [28].

The effect on serum glucose level and serum insulin level: The results present study showed that showed that the blood glucose level decreased in diabetic rats treated with Sildenafil citrate, metformin and (Sildenafil citrate \& metformin) by $48 \%, 46 \%$ and $52 \%$ respectively 
in comparison with diabetic untreated rats and serum insulin level increased by $17 \%, 142 \%$ and $154 \%$ respectively in comparison with diabetic untreated rats. In prediabetic groups there were no significant difference in serum blood glucose level of prediabetic groups as glucose level decreased by $11 \%, 11 \%$ and $17 \%$ respectively in comparison with diabetic untreated rats and serum insulin level increased by 5\%, 65\% and $67 \%$ respectively in comparison with prediabetic untreated rats as shown in fig (1). The result of the current study is in agreement with results of Ayala et al. who found that chronic inhibition of PDE-5 enhance insulin action in obese rats, which may explained by PDE-5 inhibition may improve insulin action and prevent endothelial dysfunction [29]. There is evidence supports the insulin resistance and type 2 diabetes may cussed by endothelial dysfunction [30]. Also endothelial dysfunction lead to impairing glucose uptake by muscles is by a decreasing the NO levels and cGMP production [31]. Thus, preventing a decrease in cGMP levels, resulting in improved insulin action and glucose uptake by muscles. This effect may be related to central nervous system as sildenafil has been found to cross the blood brain barrier, and PDE-5 expression has been detected in the brain [32]. Thus, signaling through cGMP in the central nervous system may play a role in the regulation of insulin action and energy homeostasis as stated by Ayala et al [29].

Hill et al observed a significant improvement in insulin sensitivity in the severely obese subjects and they suggests that this is a class effect of PDE5 inhibitors [33].

Also, these findings are agreement with El Sayed et al who found that sildenafil citrate elevated serum insulin and decreased blood glucose level in dose dependent manner and they explained it by Inhibition of cGMP hydrolysis that activates cGMP-dependent protein kinase G, which phosphorylates and activates hormone-sensitive lipase then increases free fatty acid (FFA) availability and energy expenditure [34].

The results of this study disagree with the results obtained by other workers they showed that sildenafil citrate has in significant effect in reducing the blood glucose level of diabetic rats [35-37].

The results of this study showed that, combination of metformin and sildenafil has more significant reduction $(\mathrm{P}<0.01)$ on serum blood glucose of the diabetic rats more than metformin alone or sildenafil alone but there is no significant $(\mathrm{P}<0.05)$ difference on serum blood glucose in the prediabetic treated rats in comparison with control prediabetic rats. Also, combination of metformin and sildenafil has more significant increase $(\mathrm{P}<0.01)$ on serum insulin level of the diabetic and prediabetic rats more than metformin alone but there is no significant $(\mathrm{P}<0.05)$ difference on serum insulin level between combination and sildenafil alone.

In patients with DM2, an increase in free radicals caused by various risk factors (such as dyslipidemia, obesity and hyperglycemia) might enable the oxidative stress that reduces production and availability of $\mathrm{NO}$ with the subsequent appearance of ED [38].

Inhibitors of PDE-5, such as sildenafil, require adequate levels of NO to be effective. Because IR states have decreased levels of NO and insulinsensitizing agents improve IR, we postulate that, in patients with ED and IR and a poor response to sildenafil, the addition of metformin can optimize the response to sildenafil, improving endothelial function.

We hypothesize that metformin and Sildenafil citrate improve endothelial function by reducing blood sugar levels, the present study also confirmed that metformin and Sildenafil citrate have a similar curative effect in reducing blood sugar levels, improving islet function and reducing the IR index. More ever, combination of metformin and seldinafil is more successful at improving endothelial function than Sildenafil citrate alone and metformin alone, the reason for which is currently unclear and requires further study.

The effect on lipid profile: the results of the present showed that, in diabetic rats treated with metformin, Sildenafil citrate and (Sildenafil citrate\& metformin) serum cholesterol level decreased by 53\%, 50\% and 57\% respectively in comparison with diabetic untreated rats, serum triglycerides level decreased by $50 \%, 31 \%$ and $54 \%$ respectively in comparison with diabetic untreated rats, serum HDL level increased by $37 \%, 33 \%$ and $55 \%$ respectively in comparison with diabetic untreated rats and serum LDL level decreased by $45 \%, 66 \%$ and $71 \%$ respectively in comparison with diabetic untreated rats as shown in fig (2). prediabetic rats treated with metformin, Sildenafil citrate and (Sildenafil citrate\& metformin) serum cholesterol level decreased by $37 \%, 34 \%$ and $42 \%$ respectively in comparison with prediabetic untreated rats, serum triglycerides level decreased by $44 \%$, $36 \%$ and $52 \%$ respectively in comparison with prediabetic untreated rats, serum HDL level increased by $24 \%, 19 \%$ and $35 \%$ respectively in comparison with prediabetic untreated rats and serum LDL level decreased by 34\%, 55\% and $62 \%$ respectively in comparison with diabetic untreated rats as shown in fig (2).

In the diabetic rats treated with Sildenafil citrate, our results agree with Mitschke et al. who stated that diabetic rats treated with Sildenafil citrate decreased significant of serum triglycerides, serum total cholesterol level and serum LDL level, while serum HDL level increased significant in comparison with diabetic untreated rats. This positive effect of sildenafil could be due to increased appearance of brown-like adipocytes 
in white adipose tissue [27].

The significant decrease in the serum lipid levels in treated diabetic rat might have been due to the increase insulin sensitivity following sildenafil administration.

There is an evidence that use of PDE- 5 inhibitors could overcome the oxidative stress-induced cellular dysfunctions and apoptosis associated with lipid peroxidation derivatives by increasing cyclic nucleotides [39]. Polte and Schroder, reported an antioxidant property for NO donors in vascular endothelium through concerted action of cGMP and cAMP[9]. In addition, the hypolipidemic and insulin sensitizing effects of sildenafil may be attributed to its antiinflammatory effect [39]. It was shown that sildenafil promotes browning of white adipocytes in vitro [40].

In the prediabetic rats treated with Sildenafil citrate, our results are in agreement with Ayala et al. who stated that prediabetic rats treated with Sildenafil citrate decreased significant of serum triglycerides, serum total cholesterol level and serum LDL level, while serum HDL level increased significant in comparison with prediabetic untreated rats and this improving effect of sildenafil may be explained by its direct effects of on metabolism other than eNOS activation, Improving insulin function and increase lipoprotein lipase activity[29].

Bennani-Kabchi et al explained the significant decrease in plasma TAG to the increased rate of lipolysis that is mediated by increase of plasma lipase activity [41].

The results of this study showed that, combination of metformin and sildenafil has more significant reduction $(\mathrm{P}<0.01)$ on serum cholesterol, triglycerides and LDL level of the diabetic and prediabetic rats more than metformin alone or sildenafil alone. Also, combination of metformin and sildenafil has more significant increase $(\mathrm{P}<$ 0.01 ) on serum HDL level of the diabetic and prediabetic rats more than metformin alone and sildenafil alone.

Our results are in agreement with Lizhi, who investigate the Synergistic effect of Leucine-Metformin-Sildenafil on Nonalcoholic Steatohepatitis [28].

\section{Conclusion}

Findings of the present study indicate that sildenafil citrate exerts hypoglycemic effect on diabetic albino rats. In addition to its hypoglycemic effect, it also has hypolipidemic effect on both diabetic and prediabetic albino rats and these effects may be attributed to its anti-inflammatory effect. More ever, our study revealed that combination of Sildenafil citrate and Metformin have more effect in prevention and treatment of diabetes mellitus in albino rats than treatment by Sildenafil alone or Metformin alone.

Further studies are required for further evaluation and establishment of the beneficial effects of sildenafil citrate in treatment and prevention of vascular and other complications of diabetes mellitus and mechanisms of actions.

ACKNOWLEDGEMENTS: This research received no specific grant from any funding agency in the public, commercial, or not-for-profit sectors. The authors have no potential financial conflict of interest.

Conflict of interest: The authors declare that they have no competing interest.

Financial support: This study was funded by Department of Physiology, Faculty of Medicine, Al-Azhar University, Assuit, Egypt. Without any role in the study design, recruitment of individuals, data analysis or writing of the report.

\section{REFERENCES}

1. Sanz M. Scientific evidence on the links between periodontal diseases and diabetes: Consensus report and guidelines of the joint workshop on periodontal diseases and diabetes by the International Diabetes Federation and the European Federation of Periodontology. Diabetes research and clinical practice, 2018;137:231-41

2. Zheng Y, Ley SH, Hu FB. Global aetiology and epidemiology of type 2 diabetes mellitus and its complications. Nature Reviews Endocrinology, 2018;14(2):88

3. Adi S, Gerard-Gonzalez A. Type 1 Diabetes Mellitus: An Overview, in Nutritional and Therapeutic Interventions for Diabetes and Metabolic Syndrome (2nd Edition). 2018, Elsevier. p.3-13.

4. Shah P, Trivedi NA. A meta-analysis on efficacy and tolerability of sildenafil for erectile dysfunction in patients with diabetes mellitus. Indian J. of Sexually Transmitted Diseases and AIDS. 2018;39(1): 1

5. Hackett G. British Society for Sexual Medicine Guidelines on the Management of Erectile Dysfunction in Men-2017. The J. of sexual medicine, 2018;15(4): 430-57

6. Burnett AL. Erectile Dysfunction: AUA Guideline. The Journal of urology, 2018;Sep;200(3):633-641

7. ShindelAW. Medical and surgical therapy of erectile dysfunction, in Endotext [Internet]. 2018, MDText. com, Inc.

8. Truong A. The Erectile Dysfunction Fix: How to Regain Sexual Function, Libido and Treat Erectile Dysfunction without Drugs or Surgery with Platelet Rich Plasma, the PRIAPUS SHOT®(P-SHOT®). 2018.

9. Poolsup N, Suksomboon N, Aung N. Effect of phosphodiesterase-5 inhibitors on glycemic 
control in person with type 2 diabetes mellitus: A systematic review and metaanalysis. Journal of clinical \& translational endocrinology, 2016;6: 50-5

10. Srinivasan K. Combination of high-fat diet-fed and low-dose streptozotocintreated rat: a model for type 2 diabetes and pharmacological screening. Pharmacological research, 2005;52(4):313-20

11. Saddala RR. Regulation of cardiac oxidative stress and lipid peroxidation in streptozotocin-induced diabetic rats treated with aqueous extract of Pimpinella tirupatiensis tuberous root. Experimental and toxicologic pathology. 2013;65(1-2):15-9

12. Srinivasan K. Reversal of glucose intolerance by pioglitazone in high fat diet-fed rats. Methods Find Exp Clin Pharmacol. 2004;26(5):327-33

13. Chaniotakis I. The mouse and the rat in surgical research. The anesthetic approach. Journal of the Hellenic Veterinary Medical Society. 2016;67(3): 147-62

14. Barham D, Trinder P. An improved colour reagent for the determination of blood glucose by the oxidase system. Analyst, 1972;97(1151): 142-5.

15. Lequin RM. Enzyme immunoassay (EIA)/ enzyme-linked immunosorbent assay (ELISA). Clinical chemistry. 2005;51(12): 2415-8

16. Ellefson R, Caraway W. Lipids and lipoproteins, in Fundamentals of clinical chemistry. WB Saunders Philadelphia. 1976;474-542

17. Bucolo G, David H. Quantitative determination of serum triglycerides by the use of enzymes. Clinical chemistry, 1973;19(5): 476-82

18. Duncan OD, Duncan B. A methodological analysis of segregation indexes. American sociological review, 1955;20(2):210-7.

19. Mamikutty N. The establishment of metabolic syndrome model by induction of fructose drinking water in male Wistar rats. BioMed research international. 2014; Article ID 263897, 8 pages

20. Gresser U, Gleiter C. Erectile dysfunction: comparison of efficacy and side effects of the PDE-5 inhibitors sildenafil, vardenafil and tadalafil-review of the literature. European journal of medical research, 2002;7(10):43546.

21. Nisoli E. Mitochondrial biogenesis by NO yields functionally active mitochondria in mammals. Proceedings of the National Academy of Sciences, 2004;101(47):1650712.

22. Bruckbauer A. A combination of leucine, metformin, and sildenafil treats nonalcoholic fatty liver disease and steatohepatitis in mice. International journal of hepatology, 2016

23. Tran D, Howes LG. Cardiovascular safety of sildenafil. Drug safety, 2003;26(7):453-60.

24. Das A, L. Xi, Kukreja RC. Phosphodiesterase-5 inhibitor, sildenafil preconditions adult cardiac myocytes against necrosis and apoptosis: essential role of $\mathrm{NO}$ signaling. Journal of Biological Chemistry, 2005 Apr $1 ; 280(13): 12944-55$

25. Atefi M. Alteration of glucose homeostasis by rolipram and milrinone but not sildenafil in rat primary hepatocytes culture. Prog Med Res. 2004; 2(13):1-12.

26. Kahn NN. Nitric oxide: the "second messenger" of insulin. IUBMB life, 2000;49(5): 441-50.

27. Mitschke MM. Increased cGMP promotes healthy expansion and browning of white adipose tissue. The FASEB Journal. 2013;27(4): 1621-30.

28. Fu L. Interaction between leucine and phosphodiesterase 5 inhibition in modulating insulin sensitivity and lipid metabolism. Diabetes, Metabolic syndrome and obesity: Targets and therapy, 2015;8: 227.

29. Ayala JE. Chronic treatment with sildenafil improves energy balance and insulin action in high fat-fed conscious mice. Diabetes. 2007; 56(4):1025-33.

30. Brock RW, Dorman RB. Obesity, insulin resistance and hepatic perfusion. Microcirculation. 2007; 14(4-5):339-47.

31. Sena CM, Pereira AM, Seiça R. Endothelial dysfunction-a major mediator of diabetic vascular disease. Biochimica et Biophysica Acta (BBA)-Molecular Basis of Disease. 2013;1832(12):2216-31.

32. Schultheiss D. Central effects of sildenafil (Viagra) on auditory selective attention and verbal recognition memory in humans: a study with event-related brain potentials. World journal of urology, 2001;19(1): 46-50.

33. Ho JE. Effect of phosphodiesterase inhibition on insulin resistance in obese individuals. Journal of the American Heart Association. 2014;3(5):e001001.

34. El-Sayed M.-I.K, HA-K.A. Amin. Mechanism of endothelial cyto-protective and thromboresistance effects of sildenafil, vardenafil and tadalafil in male rabbit. Archives of medical science: AMS. 2015;11(1):190.

35. Bivalacqua T. Effect of combination endothelial nitric oxide synthase gene therapy and sildenafil on erectile function in diabetic rats. International journal of impotence research. 2004;16(1): 21. 
36. Mills V. Effect of sildenafil citrate treatment in the eNOS knockout mouse model of fetal growth restriction on long-term cardiometabolic outcomes in male offspring. Pharmacological research, 2018. 137: p. 122-134.

37. Bai Y, An R. Resveratrol and sildenafil synergistically improve diabetes-associated erectile dysfunction in streptozotocininduced diabetic rats. Life sciences. 2015. 135:43-8.

38. Shukla N. Effect of sildenafil citrate and a nitric oxide donating sildenafil derivative, NCX 911 , on cavernosal relaxation and superoxide formation in hypercholesterolaemic rabbits. European journal of pharmacology.
2005;517(3): 224-31.

39. Ramirez CE. Treatment with sildenafil improves insulin sensitivity in prediabetes: a randomized, controlled trial. The Journal of Clinical Endocrinology \& Metabolism, 2015. 100(12): p. 4533-4540.

40. Lasar D. Browning attenuates murine white adipose tissue expansion during postnatal development. Biochimica et Biophysica Acta (BBA)-Molecular and Cell Biology of Lipids, 2013;1831(5): 960-8.

41. Bennani-Kabchi, N. New model of atherosclerosis in insulin resistant sand rats: hypercholesterolemia combined with D2 vitamin. Atherosclerosis, 2000;150(1): $55-61$. 